\title{
Renal Involvement in Granulomatosis With Polyangiitis Increases Economic Health Care Burden: Insights From the National Inpatient Sample Database
}

\author{
Osahon N. Idolor ${ }^{1}$, Armaan Guraya ${ }^{2}$, Chukwudi C. Muojieje ${ }^{3}$, Sandhya Shri Kannayiram ${ }^{4}$, Karun M. Nair \\ ${ }^{4}$, Jesse Odion ${ }^{5}$, Eseosa Sanwo ${ }^{6}$, Osaigbokan P. Aihie ${ }^{7}$ \\ 1. Internal Medicine, College of Medicine, University of Benin, Benin, NGA 2. College of Osteopathic Medicine, \\ Midwestern University Chicago, Chicago, USA 3. Internal Medicine, Mountain View Regional Medical Center, Las \\ Cruces, USA 4. Internal Medicine, John H. Stroger, Jr. Hospital of Cook County, Chicago, USA 5. Internal Medicine, \\ University of Benin Teaching Hospital, Benin, NGA 6. College of Medicine, University of Benin, Benin, NGA 7. School of \\ Medicine, University of Missouri, Columbia, USA
}

Corresponding author: Armaan Guraya, armaan.guraya@aol.com

\section{Abstract}

\section{Background}

This study aims to compare outcomes of hospitalizations of granulomatosis with polyangiitis (GPA) with and without renal involvement. The primary outcome was inpatient mortality, whereas secondary outcomes were hospital length of stay (LOS) and total hospital charge.

\section{Methods}

Data were abstracted from the National Inpatient Sample (NIS) 2016 and 2017 databases. The NIS was searched for GPA hospitalizations with and without renal involvement as the principal or secondary diagnosis using International Classification of Diseases, Tenth Revision, Clinical Modification (ICD-10) codes. GPA hospitalizations for adult patients from the above groups were identified. Multivariate logistic and linear regression analyses were used to adjust for possible confounders for the primary and secondary outcomes, respectively.

\section{Results}

There were more than 71 million discharges included in the combined 2016 and 2017 NIS database, of which 23,670 were for adult patients who had either a principal or secondary ICD-10 code for GPA, and 8,265 (34.92\%) of these GPA hospitalizations had renal involvement.

Review began 12/10/2020 Review ended 12/14/2020 Published 01/05/2021

\section{() Copyright 2021}

Idolor et al. This is an open access article distributed under the terms of the Creative Commons Attribution License CC-BY 4.0., which permits unrestricted use, distribution, and reproduction in any medium, provided the original author and source are credited.
Hospitalizations for GPA with renal involvement had similar inpatient mortality (3.8\% vs. 3.7\%; adjusted OR: 1.14 ; $95 \% \mathrm{CI}: 0.84-1.56$; $\mathrm{p}=0.406)$ compared to those without renal involvement. GPA with renal involvement hospitalizations had an increase in adjusted mean LOS of 1.36 days (95\% CI: 0.82-1.91; $\mathrm{p}=0.0001$ ) compared to those without renal involvement. GPA with renal involvement hospitalizations had an increase in adjusted total hospital charges of $\$ 18,723$ (95\% CI: 9,595-27,852; $\mathrm{p}=0.0001$ ) compared to those without renal involvement.

\section{Conclusions}

GPA with renal involvement hospitalizations had similar inpatient mortality compared to those without renal involvement. However, LOS and total hospital charges were greater in those with renal involvement.

Categories: Internal Medicine, Nephrology, Rheumatology

Keywords: granulomatosis with polyangiitis, renal, chronic kidney disease, vasculitis, mortality, national inpatient sample

\section{Introduction}

Granulomatosis with polyangiitis (GPA), formerly known as Wegener's granulomatosis, is a rare vasculitis affecting the small- and medium-sized vessels. It is a type of antineutrophil cytoplasmic antibody (ANCA) associated vasculitis, most commonly affecting the upper and lower respiratory tract, the kidneys, and the eyes [1]. The incidence in the United States is three per one million population, with peak incidences at 64 to 75 years of age, and recent studies have shown no sex predilection [2]. Renal involvement is very common and, together with pulmonary involvement, represents the most severe complications of the disease [3-5].

The most common renal manifestation of GPA is rapidly progressive glomerulonephritis (RPGN), leading to 
chronic kidney disease (CKD) or end-stage renal disease (ESRD) [4-6]. Renal involvement is noted in only $10-20 \%$ at presentation, but $80 \%$ of patients eventually develop renal involvement within two years of disease onset $[2,4]$. RPGN is characterized clinically by a rapid decrease in the glomerular filtration rate (GFR) of at least $50 \%$ over a short period - from a few days to three months - and histologically pauciimmune necrotizing and extensive glomerular crescent formation. Other features include microscopic hematuria, often with erythrocyte casts, and usually non-nephrotic proteinuria (less than $3 \mathrm{~g}$ ) $[1,4,7]$. Renal involvement is of particular importance because of its impact on prognosis $[2,4]$.

Most prior studies have focused on the severity and impact of renal disease in GPA in terms of long-term prognosis as well as the clinical and laboratory associations of renal involvement in GPA. Several investigators have analyzed the impact of vasculitis and demographic features at the time of diagnosis on mortality among patients with GPA. However, there are few studies elaborating on the impact of renal involvement on the overall survival in patients with GPA [8].To bridge the gap in knowledge, this study aims to compare outcomes of hospitalizations of GPA with and without renal involvement using data abstracted from the National Inpatient Sample (NIS) database.

\section{Materials And Methods}

\section{Data source}

The NIS was searched for GPA hospitalizations with and without renal involvement using International Classification of Diseases, Tenth Revision, Clinical Modification (ICD-10) codes "M31.31" and "M31.30", respectively, as the principal or secondary diagnosis. NIS is a property of the Agency for Healthcare Research and Quality. It is the largest public inpatient database in the United States of America [9-13]. NIS is a 20\% probability sampling across different strata, which is designed to be representative of all acute care hospitalizations in the US [14]. NIS maintains national representation by containing weighted discharges [15]. Each hospitalization in NIS 2016 can contain up to 30 ICD-10 diagnosis codes and 40 codes for NIS 2017. Diagnosis is either the principal diagnosis or secondary diagnosis. A principal diagnosis is the major ICD-10 code for admission. Any other diagnoses other than the principal diagnosis are secondary diagnoses [16]. This study was exempted from Institutional Review Board approval, as NIS contains de-personalized, publicly available patient data.

\section{Inclusion criteria}

We included all hospitalizations for adult patients $\geqslant 18$ years of age. We used ICD-10 codes to identify principal/secondary diagnoses. See the Appendix for a complete list of ICD-10 codes used.

\section{Outcomes}

The primary outcome was inpatient mortality. Hospital length of stay (LOS) and total hospital charges were secondary outcomes of interest.

\section{Statistical analysis}

Analyses were performed using STATA Version 16 (StataCorp, College Station, TX, USA). A univariate logistic regression analysis using all variables and co-morbidities (Table 1) was used to calculate unadjusted odds ratios (ORs) for the primary outcome. All variables with $\mathrm{p}<0.1$ were included in a multivariate logistic regression model. P-values $<0.05$ were considered significant in the multivariate analysis. Literature review was used to select confounders. Charleston index was used to control for comorbidity complexity.

Multivariate logistic and linear regression model with all variables and co-morbidities shown in Table 1 were used accordingly to adjust for confounders for the secondary outcomes.

\section{Results}

There were more than 71 million discharges included in the combined 2016 and 2017 NIS database, of which 23,670 hospitalizations were for adult patients who had either a principal or secondary ICD 10 code for GPA. Of these hospitalizations, 8,265 (34.92\%) and 15,405 (65.08\%) were for GPA with renal and without renal involvement, respectively. GPA with renal hospitalizations had less females, more secondary diagnosis of congestive heart failure (CHF), CKD, maintenance hemodialysis, anemia, first to second quartile for expected income for zip code, Medicare insured, and Charleston comorbidity score $\geqslant 3$ compared to GPA with renal involvement (Table 1).

Variables

Mean age (years)

Female
GPA $(n=23,670)$

Without Renal Involvement $(n=15,405)$

62.23

$56.48 \%$
With Renal Involvement $(n=8,265)$

$p$-Value

61.82

0.461

$47.01 \%$
$<0.0001$ 


\section{Cureus}

Race

White
Black
Hispanic
Asian
Native Americans
Others

$78.19 \%$

$74.31 \%$

$6.67 \%$

$7.77 \%$

$10.38 \%$

$11.99 \%$

$1.48 \%$

$1.77 \%$

$1.15 \%$

$1.77 \%$

$2.40 \%$

Charleston comorbidity index

0
1
2
$\geq 3$

$18.31 \%$

$7.32 \%$

$19.25 \%$

$5.38 \%$

$18.47 \%$

$24.5 \%$

$<0.0001$

$43.98 \%$

$62.79 \%$

Hospital bed size

Small

$16.52 \%$

$16.64 \%$

Medium

$27.8 \%$

$26.98 \%$

0.8511

Large

$55.66 \%$

$56.38 \%$

Hospital teaching status

Nonteaching

$28.95 \%$

$28.68 \%$

$71.32 \%$

0.8507

Teaching

$71.05 \%$

Hospital location

Rural

$7.04 \%$

$6.84 \%$

Urban

$92.96 \%$

$93.16 \%$

0.7929

Expected primary payer

\begin{tabular}{ll} 
Medicare & $62.37 \%$ \\
Medicaid & $9.18 \%$ \\
Private & $26.47 \%$ \\
Self-pay & $1.99 \%$ \\
Median household income (quartile) & \\
\hline 1st (0-25th) & $23.87 \%$ \\
\hline 2nd (26th-50th) & $25.75 \%$ \\
3rd (51st-75th) & $25.95 \%$ \\
4th (76th-100th) & $24.43 \%$
\end{tabular}

$66.67 \%$

$8.75 \%$

$22.92 \%$

0.0403

$1.66 \%$

$26.24 \%$

$28.46 \%$

$24.95 \%$

0.0085

$20.34 \%$

Hospital region

\begin{tabular}{lr} 
Northeast & $20.16 \%$ \\
Midwest & $25.22 \%$ \\
South & $35.74 \%$ \\
West & $18.89 \%$ \\
Co-morbidities & \\
\hline Dyslipidemia & $35.02 \%$ \\
Old MI & $5.71 \%$
\end{tabular}

$18.75 \%$

$25.11 \%$

$34.66 \%$

0.2247

$21.48 \%$

$33.45 \%$

0.2884

$5.69 \%$

0.9728 


\section{Cureus}

\begin{tabular}{|llll|}
\hline Atrial fibrillation/flutter & $15.35 \%$ & $16.45 \%$ & 0.3274 \\
COPD & $22.85 \%$ & $18.33 \%$ & 0.0004 \\
Carotid artery disease & $0.75 \%$ & $0.60 \%$ & 0.5778 \\
Old stroke & $6.56 \%$ & $6.35 \%$ & 0.7961 \\
Hypertension & $29.99 \%$ & $13.13 \%$ & $<0.0001$ \\
Peripheral vessel disease & $97.18 \%$ & $2.66 \%$ & 0.7493 \\
Hypothyroidism & $17.27 \%$ & $16.52 \%$ & 0.5197 \\
DM type 1 and 2 & $25.90 \%$ & $23.17 \%$ & 0.0404 \\
Obesity & $15.64 \%$ & $13.73 \%$ & 0.0890 \\
CHF & $19.15 \%$ & $25.29 \%$ & $<0.0001$ \\
CKD & $40.64 \%$ & $79.79 \%$ & $<0.0001$ \\
Liver disease & $4.35 \%$ & $3.09 \%$ & 0.0402 \\
Maintenance hemodialysis & $12.33 \%$ & $33.58 \%$ & $<0.0001$ \\
\hline O dependence & $7.59 \%$ & $5.02 \%$ & 0.0009 \\
\hline Smoking & $28.59 \%$ & $28.61 \%$ & 0.9889 \\
Anemia & $44.43 \%$ & $71.51 \%$ & $<0.0001$ \\
\hline
\end{tabular}

\section{TABLE 1: Baseline characteristics of granulomatosis with polyangiitis with and without renal involvement hospitalizations}

${ }^{*}$ Co-morbidities or secondary diagnoses

GPA, granulomatosis with polyangiitis; MI, myocardial infarction; COPD, chronic obstructive pulmonary disease; DM, diabetes mellitus; CHF, chronic congestive heart failure; CKD, chronic kidney disease; O2, oxygen; median household income, median household income for patient's Zip code

Inpatient mortality occurred in 1,010 (5.14\%) GPA hospitalizations, of which 425 (3.80\%) of the deaths occurred in GPA with renal involvement vs. 585 (3.70\%) without renal involvement. The adjusted odds ratio of inpatient mortality for GPA with renal compared to without renal involvement was 1.14 (95\% CI: 0.841.56 ; $\mathrm{p}=0.406$ ). Mean LOS of hospitalization for GPA with renal involvement was 8.14 vs. 6.59 days for GPA without renal involvement. GPA with renal involvement hospitalizations had a mean increase in adjusted mean LOS of 1.36 days (95\% CI: 0.82-1.91; $\mathrm{p}<0.0001)$ compared to GPA without renal involvement. Total hospital charge for GPA with renal involvement was $\$ 102,007$ vs. \$76,439 for GPA without renal involvement. GPA with renal involvement hospitalizations had an increase in adjusted total hospital charges by $\$ 18,723$ compared to GPA without renal involvement (95\% CI: 9,595-27,852; p<0.0001). See Table 2 for details. 


\section{Cureus}

\begin{tabular}{|c|c|c|c|c|}
\hline Primary Outcome & GPA with Renal Involvement & GPA without Renal Involvement & Adjusted Odds Ratio (95\% Cl) & p-Value \\
\hline In-hospital mortality, \% & 3.80 & 3.70 & $1.14(0.84-1.56)$ & 0.406 \\
\hline Secondary Outcomes & GPA with Renal Involvement & GPA without Renal Involvement & Adjusted Mean Difference $(95 \% \mathrm{Cl})$ & $\mathrm{p}$-Value \\
\hline LOS, mean, days & 8.14 & 6.59 & $1.36(0.82-1.91)$ & $<0.0001^{*}$ \\
\hline Total charge, mean, USD & 102,007 & 76,439 & $18,723(9,595-27,852)$ & $<0.0001^{*}$ \\
\hline \multicolumn{5}{|c|}{$\begin{array}{l}\text { TABLE 2: Clinical outcomes of granulomatosis with polyangitis hospitalizations with and without } \\
\text { renal involvement }\end{array}$} \\
\hline \multicolumn{5}{|l|}{ *Statistically significant. } \\
\hline \multicolumn{5}{|c|}{ GPA, granulomatosis with polyangiitis; LOS, hospital length of stay; USD, United States Dollars } \\
\hline
\end{tabular}

\section{Discussion}

This study compared the outcomes of hospitalizations of GPA with and without renal involvement; $35 \%$ of hospitalized patients with GPA had renal involvement, whereas $65 \%$ had no renal involvement. According to Sinico et al., renal involvement in GPA occurs only in $10-20 \%$ of cases at presentation, but $80 \%$ of patients eventually develop renal vasculitis within two years of disease onset. However, the NIS database report does not indicate when renal involvement occurred. Patients with renal involvement in this study had more baseline comorbidities (CKD, CHF, anemia, and maintenance hemodialysis). Multiple studies have shown that the principal negative predictors for GPA patient survival are age $>50$ years, dialysis dependence at presentation, high creatinine in the first month, kidney involvement (with impaired renal function), and pulmonary manifestations at diagnosis $[4,7,8,17,18]$. The identification of prognostic factors is a crucial element for the clinician in balancing the risks against the treatment benefits [7]. Therapy is associated with severe and potentially lethal adverse effects for many patients; nearly $90 \%$ of patients experience persistent morbidity despite adequate treatment [19]. Therefore, there is a need for prognostic markers of renal outcome to help to modify therapy for patients who have ANCA-associated vasculitis such as GPA [19].

RPGN, the most common renal involvement in GPA, can lead to CKD or ESRD. The classical presentation is characterized by an RPGN, macroscopic or microscopic hematuria, proteinuria, edema, decreased urine output, and rapid progressive deterioration of renal function; even with appropriate therapy, the disease may lead to chronic renal failure $[4,20,21]$. Hence, up to $30 \%$ of patients with moderate-to-severe renal disease at the time of diagnosis will require renal replacement therapy such as hemodialysis. Between $40 \%$ and $70 \%$ of patients may recover renal function following induction therapy [21]. Factors associated with return to normal renal function are unclear. However, it depends on early therapy and high doses of immunomodulators $[17,20,21]$. Our study showed that a significant number of patients with renal involvement had CKD (79.79\%) and that 34\% of GPA patients with renal involvement required maintenance dialysis compared to $12 \%$ in those without renal involvement.

The fact that renal involvement is a determinant of poor long-term prognosis was demonstrated for the first time by Carrington and Liebow [17,22]. This finding has been supported by many other studies $[4,5,17,22]$. These studies demonstrated that only those patients whose renal involvement was associated with functional impairment were at a greater risk of death. These findings suggest that the prognosis might not depend on glomerulonephritis itself but on renal function deterioration [22]. However, our study did not show a significant increase in inpatient mortality in patients with renal disease. Nationwide trends in hospitalizations and in-hospital mortality of GPA over the past two decades are mostly unknown [5,22].

Early and late-onset comorbidities significantly impact the quality of life, morbidity, and mortality rates. The latter is still unsatisfactory, and cardiovascular complications are among the principal complications leading to death [5]. The patients with renal involvement in our study had a higher percentage of congestive heart failure (25.29\%) compared to those without renal involvement (19.15\%). GPA has been suggested to be a strong independent risk factor for heart failure, although scarce data exist regarding the prevalence of cardiovascular risk factors and outcomes, including heart failure. Cardiac involvement was first described by Wegner in 1936, and since then it has been reported in 6-44\% of patients diagnosed with GPA, with the latter attributed to those with greater disease severity [19,23]. Alongside coronary arteritis, pericarditis is the most common cardiac manifestation (accounting for around $50 \%$ of all cardiac cases). Other cardiac presentations include ischemia, heart failure, valvular disorders, conduction abnormalities, and myocarditis [19,23].

We saw a significant increase in the mean length of hospital stay of 8.14 days for GPA with renal involvement compared to 6.59 days for GPA without renal involvement. GPA with renal involvement hospitalizations had an increase in adjusted mean LOS of 1.36 days (95\% CI: 0.82-1.91; p<0.0001) compared 
to GPA without renal involvement. This is an interesting finding. There are no studies known to the authors to compare or contrast this finding. However, the study conducted by Luo et al., which investigated the 30day all-cause hospital readmissions in patients with GPA using data from the 2014 National Readmission Database (NRD), a U.S.-based nationwide all-payer hospital inpatient database, is worthy of note. GPA readmissions were associated with higher LOS ( 8.0 vs. 7.2 days; $p=0.019)$ and less discharge home (50\% vs $63 \%, p<0.001$ ) [24]. Hence GPA admissions with renal involvement and GPA readmissions prolong hospital LOS compare to GPA admissions without renal involvement and index GPA hospitalizations. The prolonged LOS in GPA with renal involvement admissions may indicate a higher level of complexity in these patients, which causes increased health care expenditure.

GPA with renal involvement hospitalizations had an increase in adjusted total hospital charges of $\$ 18,723$ compared to GPA without renal involvement (95\% CI: 9,595-27,852; p<0.0001). Studies available only compared hospitalization cost and charges of GPA hospitalizations vs. non-GPA hospitalizations. GPA hospitalizations were associated with higher healthcare expenditure as demonstrated by increased adjusted mean total hospital cost of $\$ 5,125$ (95\% CI: 4,719-5,531) and adjusted mean total hospital charges of $\$ 16,841$ (95\% CI: 15,280-18,403) compared to hospitalizations without GPA [25].

The large sample size that increases the study power is the major strength of our study. However, our study has some limitations. NIS uses claims data based on ICD-10 codes, which were created for billing purposes [26]. ICD-10 codes do not grade severity [27]; therefore, we cannot discern if GPA disease severity may have had affected outcomes. NIS database contains reports on hospitalizations rather than individual patients [28]. Data on medication compliance is not available in the NIS [29,30]. NIS does not contain information on the time of diagnosis and duration of renal involvement in GPA patients.

\section{Conclusions}

There is no statistically significant difference in inpatient mortality for hospitalizations of GPA with and without renal involvement. However, LOS and total hospital charge in GPA with renal involvement were greater than those without renal involvement. Hence, GPA with renal involvement has a greater burden to the healthcare system compared to without renal involvement.

\section{Appendices}




\section{Cureus}

\section{ICD-10 codes}

\section{Diagnosis codes}

GPA with renal involvement

M31.31

GPA without renal involvement

M31.30

Comorbidities

Dyslipidemia

E78

Old MI

1252

Atrial fibrillation/flutter

148

COPD

J41, J42, J43, J44

Carotid artery disease

1652

Old stroke

163

Hypertension

110

Peripheral vascular disease

1739

Hypothyroidism

E03

DM type 1 and 2

E10, E11

Obesity

E660, E6601, E6609, E661, E662, E668, E669

Congestive heart failure

150

CKD

N18

Liver disease

K70, K71, K72, K73, K74, K75, K76, K77

Maintenance dialysis

Z992

$\mathrm{O}_{2}$ dependence

Z9981

Smoking

Z87891, F17200

Anemia

D50, D51, D52, D53, D55, D56, D57, D58, D59, D60, D61, D62, D63, D64

\section{TABLE 3: Supplementary table of used ICD-10 codes}

ICD-10, International Classification of Diseases, Tenth Revision; GPA: granulomatosis with polyangiitis, MI: myocardial infarction, COPD, chronic obstructive pulmonary disease, DM: diabetes mellitus, CKD, chronic kidney disease, $\mathrm{O}_{2}$, oxygen

\section{Additional Information \\ Disclosures}

Human subjects: Consent was obtained by all participants in this study. N/A issued approval N/A. This study was exempted from Institutional review board approval since NIS contains de-personalized, publicly available patient data. Animal subjects: All authors have confirmed that this study did not involve animal subjects or tissue. Conflicts of interest: In compliance with the ICMJE uniform disclosure form, all authors declare the following: Payment/services info: All authors have declared that no financial support was received from any organization for the submitted work. Financial relationships: All authors have declared that they have no financial relationships at present or within the previous three years with any organizations that might have an interest in the submitted work. Other relationships: All authors have declared that there are no other relationships or activities that could appear to have influenced the submitted work.

\section{References}

1. Grygiel-Górniak B, Limphaibool N, Perkowska K, Puszczewicz M: Clinical manifestations of granulomatosis with polyangiitis: key considerations and major features. Postgrad Med. 2018, 130:581-596. 10.1080/00325481.2018.1503920 
2. Garlapati P, Qurie A: Granulomatosis with polyangiitis. StatPearls [Internet]. StatPearls Publishing, Treasure Island, FL; 2020.

3. Wallace ZS, Lu N, Miloslavsky E, Unizony S, Stone JH, Choi HK: Nationwide Trends in hospitalizations and in-hospital mortality in granulomatosis with polyangiitis (Wegener's). Arthritis Care Res. 2017, 69:915-21. 10.1002/acr.22976

4. Sinico RA, Di Toma L, Radice A: Renal involvement in anti-neutrophil cytoplasmic autoantibody associated vasculitis. Autoimmun Rev. 2013, 12:477-482. 10.1016/j.autrev.2012.08.006

5. Kronbichler A, Shin J Il, Lee KH, et al.: Clinical associations of renal involvement in ANCA-associated vasculitis. Autoimmun Rev. 2020, 19:102495. 10.1016/j.autrev.2020.102495

6. Aslam MI, Awan AUR, Haider AS: Determine the clinical pattern of anti-neutrophil cytoplasmic antibody associated vasculitis and examine the long term prognostic factors of patients and renal survival and relapse. Pakistan J Med Heal Sci. 2019, 13:998-1000.

7. Binda V, Moroni G, Messa P: ANCA-associated vasculitis with renal involvement. J Nephrol. 2018, 31:197208. 10.1007/s40620-017-0412-z

8. Arslan E, Oruc M, Yalin SF, et al.: Clinical features and outcomes of patients with granulomatosis with polyangiitis and review of literature. Turkish J Nephrol. 2020, 29:279-288. 10.5152/turkjnephrol.2020.4048

9. Edigin E, Prado V, Shaka H, Salazar M: Lung involvement in systemic lupus erythematosus increases inpatient mortality: analysis of the National Inpatient Sample. Chest. 2020, 4:1871.

10.1016/j.chest.2020.08.1623

10. Edigin E, Shaka H, Xu J, Eseaton PO, Ojemolon PE, Asemota IR, Akuna E: Diabetes mellitus does not negatively impact hospitalizations for systemic sclerosis. Am Heart J. 2020, 229:161. 10.1016/j.ahj.2020.10.013

11. Asemota I, Shaka H, Edigin E, et al.: Co-existing protein energy malnutrition is associated with increased mortality in patients admitted for transcatheter aortic valve replacement: analysis of the National Inpatient Sample. JACC. 2020, 76:34-35.

12. Edigin E, Shaka H, Eseaton PO, Ojemolon PE, Akuna E, Asemota IR: Do rheumatoid arthritis patients have worse outcomes when hospitalized for heart failure exacerbation?. Am Heart J. 2020, 229:160. 10.1016/j.ahj.2020.10.011

13. Shaka H, Edigin E, Gomez TMA, Eseaton PO: Diabetic ketoacidosis is one of the major reasons for hospitalizations among type 1 diabetics which led to inpatient mortality. Findings from the Nationwide Inpatient Sample. Am Heart J. 2020, 229:159-160. 10.1016/j.ahj.2020.10.010

14. Ojemolon PE, Shaka H, Edigin E, et al.: Impact of diabetes mellitus on outcomes of patients with knee osteoarthritis who underwent knee arthroplasty: an analysis of the Nationwide Inpatient Sample. Cureus. 2020, 12:8902. 10.7759/cureus.8902

15. Edigin E, Shaka H, Eseaton P, et al.: Rheumatoid arthritis is not associated with increased inpatient mortality in patients admitted for acute coronary syndrome. Cureus. 2020, 12:9799. 10.7759/cureus.9799

16. Edigin E, Ojemolon PE, Eseaton PO, et al.: Rheumatoid arthritis patients have better outcomes when hospitalized for ischemic stroke: analysis of the National Inpatient Sample [Online ahead of print]. J Clin Rheumatol. 2020, 10.1097/RHU.0000000000001563

17. Mahr A, Girard T, Agher R, Guillevin L.: Analysis of factors predictive of survival based on 49 patients with systemic Wegener's granulomatosis and prospective follow-up. Rheumatology. 2001, 40:492-498. 10.1093/rheumatology/40.5.492

18. Koldingsnes W, Nossent JC: Baseline features and initial treatment as predictors of remission and relapse in Wegener's granulomatosis. J Rheumatol. 2003, 30:80-88.

19. de Lind van Wijngaarden RA, Hauer HA, Wolterbeek R, et al.: Clinical and histologic determinants of renal outcome in ANCA-associated vasculitis: a prospective analysis of 100 patients with severe renal involvement. J Am Soc Nephrol. 2006, 17:2264-2274. 10.1681/ASN.2005080870

20. Renaudineau Y, Le Meur Y: Renal involvement in Wegener's granulomatosis. Clin Rev Allergy Immunol. 2008, 35:22-29. 10.1007/s12016-007-8066-6

21. José F, Gilberto J, Rodolfo T, et al.: Renal compromise in a patient with Wegener's granulomatosis: clinical case and literature review. Revista Colombiana Nefrología. 2017, 4:99-111. 10.22265/acnef.4.1.234

22. Şen N, Aydin M, Yildiz R, et al.: Granulomatous polyangitis (Wegener granulomatosis): clinical findings and results of long-term follow-up. Tuberk Toraks. 2016, 64:223-229. 10.5578/tt.25269

23. Parmar MK, Alikhan M, Hsu VM, Borham A: Echocardiogram: the GPS to GPA's Heart (granulomatosis with polyangiitis). Case Rep Rheumatol. 2019, 2019:1-3. 10.1155/2019/7609386

24. Luo Y, Jiang C, Arevalo Molina AB, Murray S, Anez GC, Salgado M, Xu J: Thirty-day hospital readmissions for granulomatosis with polyangiitis in the United States: a nationwide analysis. J Clin Rheumatol. 2020, 26:192-196. 10.1097/RHU.0000000000001045

25. Quartuccio L, Treppo E, Valent F, De Vita S: Healthcare and economic burden of ANCA-associated vasculitis in Italy: an integrated analysis from clinical and administrative databases [Online ahead of print]. Intern Emerg Med. 2020, 10.1007/s11739-020-02431-y

26. Jamal S, Khan MZ, Kichloo A, et al.: The effect of atrial fibrillation on inpatient outcomes of patients with acute pancreatitis: a two-year National Inpatient Sample database study. J Innov Cardiac Rhythm Manage. 2021, 12:1-6.

27. Edigin E, Ojemolon PE, Eseaton PO, Shaka H, Akuna E, Asemota IR, Manadan A: Systemic sclerosis is associated with increased inpatient mortality in patients admitted for atrial fibrillation: analysis of the National Inpatient Sample [Online ahead of print]. J Clin Rheumatol. 2020, 10.1097/RHU.0000000000001543

28. Edigin E, Kaul S, Eseaton PO: Analysis of hidradenitis suppurativa hospitalizations: a report from the National Inpatient Sample database [Online ahead of print]. J Am Acad Dermatol. 2020, 10.1016/j.jaad.2020.10.083

29. Edigin E, Akuna E, Asemota I, Eseaton P, Ojemolon PE, Shaka H, Manadan A: Rheumatoid arthritis does not negatively impact outcomes of patients admitted for atrial fibrillation. Cureus. 2020, 12:10241. 10.7759/cureus.10241

30. Edigin E, Ojemolon PE, Eseaton PO, et al.: Systemic sclerosis is associated with increased inpatient mortality 


\section{Cureus}

in patients admitted for acute coronary syndrome: analysis of the National Inpatient Sample [Online ahead of print]. J Clin Rheumatol. 2020, 10.1097/RHU.0000000000001634 